EXTENDED REPORT

\title{
A comparison of outcomes after indocyanine green and trypan blue assisted internal limiting membrane peeling during macular hole surgery
}

\author{
K L Lee, S Dean, S Guest
}

Br J Ophthalmol 2005;89:420-424. doi: 10.1136/bjo.2004.049684

\begin{abstract}
See end of article for authors' affiliations

.....................

Correspondence to: Mr Stephen Guest, Department of Ophthalmology, Waikato Hospital, Hamilton, New Zealand; s.guest@clear. net.nz

Accepted for publication 1 August 2004
\end{abstract}

\begin{abstract}
Aims: To compare the anatomical and visual outcomes of macular hole repair surgery using indocyanine green (ICG) or trypan blue (TB) staining of the internal limiting membrane (ILM).

Method: Retrospective analysis of 37 eyes from 37 consecutive patients with stage 2, 3, and 4 idiopathic macular holes who underwent macular hole repair by one surgeon using the same technique but utilising different dyes for ILM peeling. In 19 patients ICG was used while 18 patients had TB. The anatomical and visual results in these two groups were compared.

Results: There were no significant differences in the demographic and macular hole characteristics of the ICG and TB groups. Macular hole closure was achieved in $91.9 \%$ of all patients of which the ICG group had an $89.5 \%$ hole closure rate and the TB group had a $94.4 \%$ hole closure rate. After excluding cases with failed hole closure and other vision affecting complications, there was no significant difference between the preoperative visual acuities in the TB and ICG groups but the postoperative visual acuities were better in the TB than the ICG group $(p=0.036)$. The TB group also had more Snellen lines of improvement than the ICG group (2.94 $\vee 1.79$ lines; $p=0.046$ ).

Conclusion: TB appears to be less toxic than ICG when used in dye assisted peeling of ILM during macular hole repair as reflected by the better visual results in the TB group of patients.
\end{abstract}

$\mathrm{T}$ he successful closure of macular hole by pars plana vitrectomy and fluid-air exchange has revolutionised the treatment of this previously untreatable condition. Peeling of the nearly invisible internal limiting membrane (ILM) of the retina has been advocated by many as it may improve the postoperative anatomical and functional visual outcome of macular hole surgery. ${ }^{1-4}$ This is a technically challenging procedure because the ILM is difficult to grasp and the extent of the peel is difficult to delineate. This problem appeared to have been solved by the arrival of indocyanine green (ICG) as a vital dye in vitreoretinal surgery. ICG staining of ILM made peeling faster, and possibly less traumatic than without dye, but there has been considerable debate recently regarding its potential toxicity. ${ }^{56}$ There are reports of retinal elements such as Muller cell plasma membrane on histopathological sections of ILM specimens after ICG assisted ILM peeling. ${ }^{78}$ Gandorfer et al have interpreted this as an alteration of the cleavage plane from the ILM to the innermost retinal layers. ${ }^{8}$ Ho et al found that cultured human retinal pigment epithelium (RPE) cells exposed to ICG with concentrations up to $5 \mathrm{mg} / \mathrm{ml}$ for up to 5 minutes were not injured but prolonged exposure to low dose ICG was toxic to cultured RPE cells. ${ }^{9}$ This finding may be significant as ICG autofluorescence in the posterior segment has been shown to be present for up to 9 months after macular hole repair with ICG assisted ILM peeling. This could also lead to delayed photochemical damage to the RPE. ${ }^{10-13}$ Stalmans et al reported that the toxic effect of ICG on RPE might be related to the hypo-osmolarity of the solvent but this view is not universally shared. ${ }^{14}{ }^{15}$ The advent of trypan blue (TB) as a second generation vital stain in vitreoretinal surgery may address some of these issues. ${ }^{16} \mathrm{~TB}$ directly stains epiretinal membrane (ERM) and to a lesser extent ILM, making it useful for both macular hole and macular pucker surgery. ${ }^{17-19}$ TB concentrations of $0.15 \%$ or less have been reported to exert minimal toxic effects at the neurosensory retina following a contact time of 1 month in an animal model. ${ }^{20}$ Short exposure of TB with concentrations of up to $0.3 \%$ were not found to have a toxic effect on cultured RPE cells. ${ }^{21}$ The purpose of this study is to compare the anatomical and visual outcomes of macular hole surgery using ICG or TB.

\section{MATERIALS AND METHODS}

Thirty seven eyes of 37 consecutive patients with stage 2,3 , and 4 idiopathic macular holes underwent macular hole repair by one surgeon ( $S G$ ) using the same technique on all patients, but using different dyes (ICG or TB) for ILM peel (table 1). ICG was used in 19 cases and TB in 18 cases. There was no selection bias in the choice of dye used. ICG was used in the initial cases but as TB became available, it became the dye of choice.

The surgical technique involved lens extraction by phacoemulsification and posterior chamber intraocular lens insertion (except for one patient who was already pseudophakic), followed by three port pars plana vitrectomy. Posterior vitreous detachment was induced where necessary and any visible epiretinal membrane was removed with forceps. This was followed by dye assisted ILM peeling in a continuous curvilinear fashion leaving a circular ILM defect with a radius of 2 disc diameters centred on the macular hole. A standard Accurus illumination probe (Alcon Laboratories, Fort Worth, TX, USA) was used for illumination (Hi2 setting). All patients had gas tamponade and were advised to position prone for 1 week (in 36 cases $15 \% \mathrm{C}_{3} \mathrm{~F}_{8}$ was used and in one case $20 \%$ $\mathrm{SF}_{6}$ was used).

In the ICG group, $0.5 \%$ solutions were used in the initial patients followed in later patients by $0.05 \%$ solution. $25 \mathrm{mg}$

Abbreviations: BSS, balanced salt solution; ERM, epiretinal membrane; ICG, indocyanine green; ILM, internal limiting membrane; RPE, retinal pigment epithelium; TB, trypan blue 


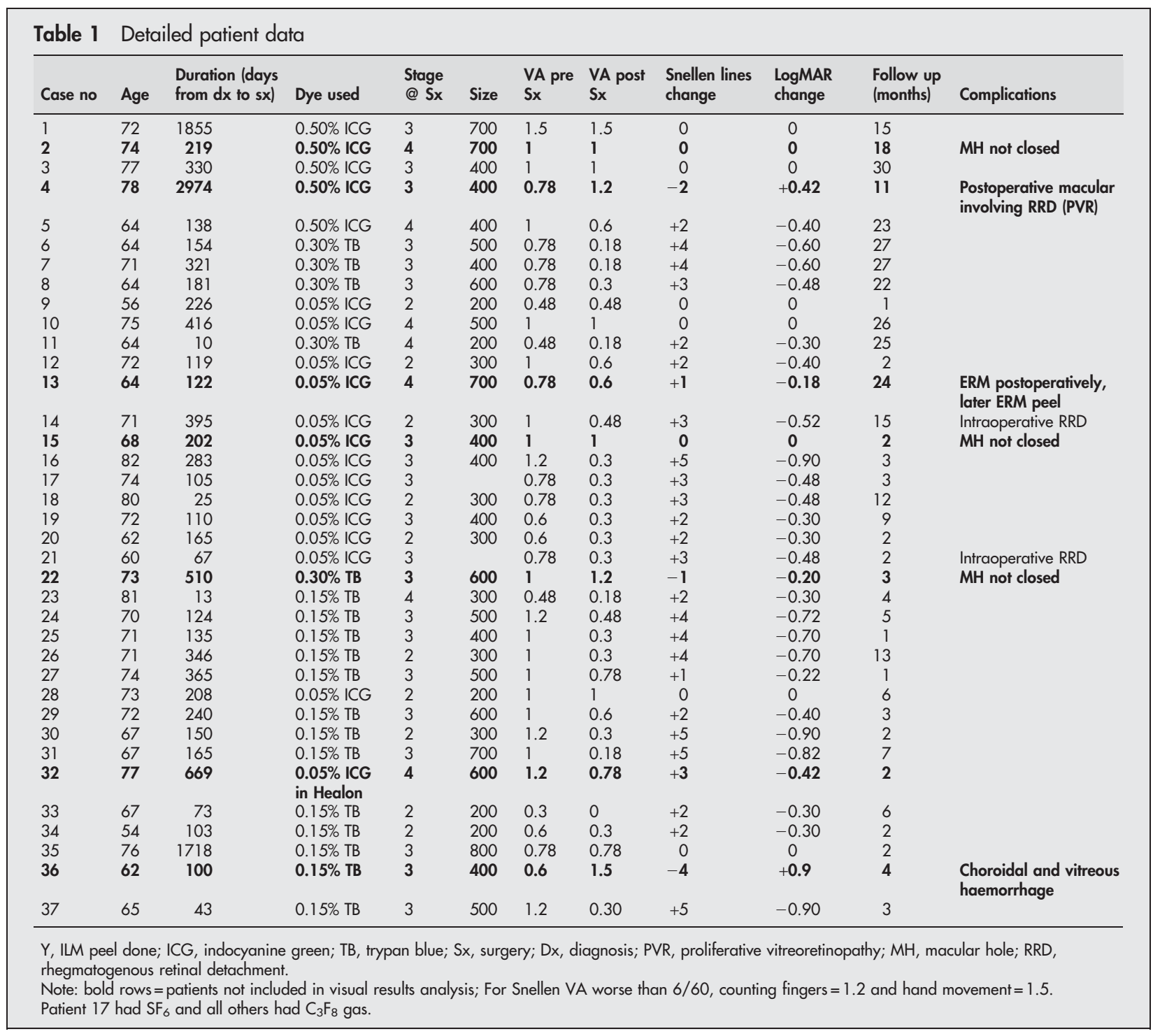

vials of ICG (IC-Green, Akorn, Buffalo Grove, IL, USA) were reconstituted with $1 \mathrm{ml}$ of supplied diluent (Aqueous Solvent) and $4 \mathrm{ml}$ of balanced salt solution (BSS, Alcon Laboratories, Fort Worth, TX, USA) to obtain a $0.5 \%$ ICG solution. A volume of $1 \mathrm{ml}$ of this solution was mixed with $9 \mathrm{ml}$ of BSS to obtain a $0.05 \%$ ICG solution. A $5 \mu \mathrm{m}$ and $0.2 \mu \mathrm{m}$ filter were used when removing the dye from the original vial. The ICG solution was given to the surgeon in a $2 \mathrm{ml}$ syringe with Rycroft needle. Approximately $0.5 \mathrm{ml}$ of the dye was injected over the macula and then aspirated immediately so that the vitreous cavity was cleared of dye between 30 and 60 seconds after injection. An MVR blade was used to start the ILM peel, which was finished with forceps.

In the TB group, $0.3 \%$ solutions were used in the first five patients with the remaining patients having commercially available $0.15 \%$ TB solution (MembraneBlue, DORC International bv, Zuidland, Netherlands). In the initial cases, following vitrectomy, fluid-air exchange was performed before injection of TB and the dye was aspirated under air. More recently, the technique was modified to direct application of the dye over the macula in a fluid filled posterior segment with immediate aspiration. ILM peeling was performed using the technique described above.
Sample osmolarities of the $0.50 \%$ and $0.05 \%$ ICG dye solutions and the commercially available $0.15 \%$ TB solution were measured by our hospital biochemistry laboratory.

The patient demographics and individual clinical features were recorded retrospectively. The anatomical outcome measured was the rate of macular hole closure postoperatively. All complications were noted for each group and visual outcomes were measured and compared for ICG and TB groups. The gradations on the Snellen visual acuity charts used were $6 / 6,6 / 9,6 / 12,6 / 18,6 / 24,6 / 36$, and 6/60, followed by counting fingers and hand movement.

Two tailed $t$ tests were conducted for analyses of continuous variables between the two groups. The variables included preoperative and postoperative logMAR BCVA, duration of symptoms, duration of follow up, size of macular hole and mean lines of BCVA improvement.

\section{RESULTS}

Thirty seven eyes of 37 patients from a consecutive series were included in the anatomical outcome analysis. Of these 37 patients, 19 underwent ILM peeling using ICG and 18 had TB.

Visual results for these groups were compared after exclusion of patients in whom the postoperative visual 


\begin{tabular}{|c|c|c|c|c|}
\hline Parameters (averages) & Total $(n=30)$ & ICG $(n=14)$ & TB $(n=16)$ & ICG $v$ TB ( $t$ test) \\
\hline Age (years) (range) & $69.6(54-82)$ & $70.7(56-82)$ & $68.6(54-81)$ & $p=0.411$ \\
\hline $\begin{array}{l}\text { Number of female patients } \\
(\%)\end{array}$ & $22(73.3 \%)$ & $10(71.4 \%)$ & $12(75.0 \%)$ & \\
\hline $\begin{array}{l}\text { Duration between diagnosis } \\
\text { and surgery (days) (range) }\end{array}$ & $286.1(10-1855)$ & $317.3(25-1855)$ & $258.8(10-1718)$ & $p=0.713$ \\
\hline $\begin{array}{l}\text { Follow up postop (months) } \\
\text { (range) }\end{array}$ & $10.0(1-30)$ & $10.6(1-30)$ & $9.4(1-27)$ & $p=0.729$ \\
\hline \multicolumn{5}{|l|}{ Stage of holes (number; \%): } \\
\hline Stage 2 & $9(30.0 \%)$ & $6(42.9 \%)$ & $3(18.8 \%)$ & \\
\hline Stage 3 & $16(53.3 \%)$ & $6(42.9 \%)$ & $10(66.6 \%)$ & \\
\hline Stage 4 & $5(16.7 \%)$ & $2(14.2 \%)$ & $3(18.8 \%)$ & \\
\hline $\begin{array}{l}\text { Average size of macular } \\
\text { holes }(\mu \mathrm{m}) \text { (range) }\end{array}$ & $407.1(200-700)$ & $366.7^{*}(200-700)$ & $437.5(200-800)$ & $p=0.270$ \\
\hline
\end{tabular}

acuities may have been affected by non-dye related factors such as persistent macular holes postoperatively (cases 2, 15, and 22), macula involving rhegmatogenous retinal detachment (case 4), postoperative macular epiretinal membrane requiring removal (case 13) and a patient with peripheral choroidal haemorrhage postoperatively together with persistent vitreous cavity haemorrhage (case 36). The occurrence of epiretinal membrane following ILM peeling is very unusual and was unexpected. Case 32 was also excluded from the visual analysis because a mixture of ICG and viscoelastic was used to stain only half of the macula. This was the only patient in the series on whom this was tried. After these exclusions, the comparison of preoperative variables and postoperative visual outcomes was based on 14 of 19 ICG patients and 16 of $18 \mathrm{~TB}$ patients (that is, 30 patients in total) (tables 2 and 3 ).

\section{Demographic and clinical features (table 2)}

The average age in the TB group was 2 years less than in the ICG group and most patients in both groups were female. The duration of symptoms before surgery and the length of follow up postoperatively were both a little longer for the ICG group than the TB group, but not significantly so. Follow up was as short as 1 month in three patients (one ICG and two TB patients), which was largely because of patient discharge to remote areas where patient tracking was difficult. There was also a tendency for the ICG group to have smaller diameter macular holes, although again there was no significant difference. The ICG group also had proportionally more stage 2 than stages 3 or 4 macular holes compared to the TB group.

\section{Anatomical results and complications (table 1)}

Of the 37 patients who underwent dye assisted ILM peeling, 34 had successful macular hole closure (91.9\%). The ICG group had $89.5 \%$ rate of macular hole closure postoperatively ( 17 of 19 eyes) compared to a $94.4 \%$ closure rate for the TB group (17 of 18 eyes). Three eyes had persistent macular holes postoperatively and these patients elected not to have further repair. One patient (case 4) had a postoperative macula involving rhegmatogenous retinal detachment associated with proliferative vitreoretinopathy and required silicone oil and a circumferential buckle. The retina remained attached but the final vision was poor. Two other patients had small macula on rhegmatogenous retinal detachments intraoperatively which were repaired at the time (cases 14 and 21) and did not affect the postoperative vision. One case with choroidal haemorrhages and another with epiretinal membrane development have already been mentioned.

\section{Visual results (table 3 )}

The visual results of 14 ICG patients were compared with 16 patients on whom TB was used. There was no statistically significant difference between the preoperative $\log$ MAR visual acuities of the two groups, but there was a significant difference in the postoperative $\log$ MAR visual acuities, with the TB group having better postoperative visual acuities than the ICG patients $(p=0.036)$. As might be expected, both groups showed a statistically significant improvement in $\log$ MAR vision after surgery $(\mathrm{p}=0.020$ for ICG $v 0.000005$ for $\mathrm{TB}$ ). The difference between preoperative and postoperative $\log$ MAR visual acuity was calculated for each patient and the ICG and TB group figures compared. There was no statistically significant difference between the two groups $(p=0.053)$. However, the average number of lines of improvement on the Snellen chart in the TB group was greater than that in the ICG group (2.94 lines $v 1.79$ lines) and this difference was statistically significant $(p=0.046)$. In the ICG group, there were only three patients who had the

Table 3 Visual acuity outcomes

\begin{tabular}{|c|c|c|c|c|}
\hline Parameters (averages) & Total $(n=30)$ & ICG $(n=14)$ & TB $(n=16)$ & $\begin{array}{l}\text { ICG } v \text { TB } \\
(t \text { test) }\end{array}$ \\
\hline \multicolumn{5}{|l|}{ Preoperative VA } \\
\hline LogMAR (range) & $0.88(0.3-1.5)$ & $0.91(0.6-1.5)$ & $0.85(0.3-1.2)$ & \multirow[t]{2}{*}{$p=0.550$} \\
\hline Snellen (range) & $6 / 46(6 / 12-6 / 190)$ & $6 / 49(6 / 24-6 / 190)$ & $6 / 42(6 / 12-6 / 92)$ & \\
\hline \multicolumn{5}{|l|}{ Postoperative VA } \\
\hline LogMAR (range) & $0.46(0-1.5)$ & $0.60(0.3-1.5)$ & $0.36(0-0.78)$ & \multirow[t]{2}{*}{$p=0.036$} \\
\hline Snellen (range) & $6 / 18(6 / 6-6 / 190)$ & $6 / 24(6 / 12-6 / 190)$ & $6 / 14(6 / 6-6 / 36)$ & \\
\hline $\begin{array}{l}\text { Change in VA (logMAR) } \\
\text { (range) }\end{array}$ & $0.42(0-0.90)$ & $0.30(0-0.90)$ & $0.50(0-0.90)$ & $p=0.053$ \\
\hline $\begin{array}{l}\text { Snellen VA lines of } \\
\text { improvement (range) }\end{array}$ & $2.40(0-5)$ & $1.79(0-5)$ & $2.94(0-5)$ & \multirow[t]{2}{*}{$p=0.046$} \\
\hline $\begin{array}{l}\text { VA } 6 / 12 \text { or better } \\
\text { postoperatively (number; \%) }\end{array}$ & $17(56.7 \%)$ & $6(42.9 \%)$ & $11(61.1 \%)$ & \\
\hline
\end{tabular}


$0.50 \%$ concentration and there was no statistical difference in the amount of visual improvement seen in this small group when compared to the other 11 patients who had the $0.05 \%$ concentration. In the TB group, only four patients had the $0.30 \%$ concentration compared to 12 who had the $0.15 \%$ concentration and, again, there was no significant difference in the amount of visual improvement. In those eyes that had a minimum of 6 months follow up, the TB patients had a greater improvement in logMAR VA postoperatively than those having ICG $(p=0.019)$ and more Snellen lines were gained $(\mathrm{p}=0.006)$.

\section{Dye osmolarities}

The $0.50 \%$ ICG had an osmolarity of $242 \mathrm{mOsm} / \mathrm{kg}$ while the $0.05 \%$ ICG had an osmolarity of $295 \mathrm{mOsm} / \mathrm{kg}$ reflecting the greater proportion of saline (as opposed to aqueous) diluent used in the lower concentration dye solution. The $0.15 \% \mathrm{~TB}$ had an osmolarity of $293 \mathrm{mOsm} / \mathrm{kg}$.

\section{DISCUSSION}

Some authors have reported no significant improvement in vision following ICG assisted ILM peeling in macular hole repair when compared with preoperative visual acuities ${ }^{22}$ or macular hole surgery without ILM peeling. ${ }^{23}$ Ando et al found no significant improvement in visual acuity in a group of patients having ICG assisted macular hole surgery, whereas they report of another group having ILM peeling without ICG dye that did improve postoperatively. ${ }^{24}$ In light of the possible toxic effects of ICG on the RPE and neural retina, TB is being used more frequently as an alternative dye in vitreomacular surgery. Whereas ICG selectively stains the acellular ILM, ${ }^{25}$ TB stains cellular structures such as epiretinal membrane, but also, to a lesser degree, the ILM. ${ }^{17-19}$ The application of TB onto the ILM or ERM results in good visualisation scores and ease of removal scores. ${ }^{18}$ In our experience, ICG stains the ILM better than TB although peeling of the ILM is still significantly facilitated by the use of TB. There have been a few preliminary reports of TB assisted ERM and ILM peeling in vitreoretinal surgery for proliferative vitreoretinopathy, idiopathic ERM, and macular hole with satisfactory anatomical, and functional results. ${ }^{17} 2627$ To our knowledge, there have been no other reports comparing the effectiveness of macular hole repair using TB and ICG.

In this study, macular hole closure was successful in $91.9 \%$ of all patients who underwent dye assisted ILM peeling. These rates are comparable to previous studies where macular hole repair was performed with ILM peeling with or without dye assistance. ${ }^{12232628-32}$ Patients who had undergone ILM peeling using TB achieved better postoperative visual acuities and greater lines of improvement on the Snellen visual acuity chart than patients in whom ICG had been used. The ICG group had slightly more longstanding holes in older patients, but the TB patients had larger holes of later stage and follow up was a little shorter. The better visual results of the TB patients may reflect the toxicity of ICG, but could simply be due to chance.

This is a retrospective study with several flaws. The patient numbers are low and the follow up period short in many cases. Acuities were not measured with ETDRS charts and varying concentrations of ICG and TB were used. In the study's favour is the fact that all operations were performed by a single surgeon, eliminating individual variability, and all the patients were pseudophakic postoperatively thereby eliminating the effect of cataract progression on the postoperative visual acuity measures.

The awareness of possible ICG toxicity provided the impetus for us to start using $\mathrm{TB}$ for vitreomacular surgery in our institution. TB may have a better safety profile based on our current knowledge. The use of TB in ILM peeling for macular hole repair appears to give anatomical and visual results, that are at least as good as, if not better than those after ICG assisted surgery. TB has the potential to replace ICG as the first choice dye for vitreomacular surgery but more work needs to be done to establish the safety profile of both dyes.

\section{Authors' affiliations}

\section{K L Lee, S Dean, S Guest, Department of Ophthalmology, Waikato} Hospital, Hamilton, New Zealand

The authors have no financial interest in any of the products mentioned in the study and have not received any funding from the manufacturers.

\section{REFERENCES}

1 Park DW, Sipperley JO, Sneed SR, et al. Macular hole surgery with internallimiting membrane peeling and intravitreous air. Ophthalmology 1999; 106:1392-8

2 Brooks HL Jr. Macular hole surgery with and without internal limiting membrane peeling. Ophthalmology 2000;107:1939-49.

3 Haritoglou C, Gass CA, Schaumberger M, et al. Long-term follow-up after macular hole surgery with internal limiting membrane peeling. Am J Ophthalmol 2002;134:661-6.

4 Mester V, Kuhn F. Internal limiting membrane removal in the management of full-thickness macular holes. Am J Ophthalmol 2000;129:769-77.

5 Kampik A, Sternberg P. Indocyanine green in vitreomacular surgery-(why) is it a problem? Am J Ophthalmol 2003;136:527-9.

6 Sebag J. Indocyanine green-assisted macular hole surgery: too pioneering? Am J Ophthalmol 2004; 137:744-6

7 Kwok AKH, Li WWY, Pang CP, et al. Indocyanine green staining and removal of the internal limiting membrane in macular hole surgery: histology and outcome. Am J Ophthalmol 2001;132:178-83.

8 Gandorfer A, Haritoglou C, Gass CA, et al. Indocyanine green-assisted peeling of the internal limiting membrane may cause retinal damage. Am J Ophthalmol 2001;132:431-3.

9 Ho JD, Tsai RJF, Chen SN, et al. Cytotoxicity of indocyanine green on retinal pigment epithelium: implications for macular hole surgery. Arch Ophthalmol 2003;121:1423-9.

10 Ashikari M, Ozeki H, Tomida K, et al. Retention of dye after indocyanine green-assisted internal limiting membrane peeling. Am J Ophthalmol 2003; 136:172-4

11 Ciardella AP, Schiff W, Barile G, et al. Persistent indocyanine green fluorescence after vitrectomy for macular hole. Am J Ophthalmol 2003; 136:174-7

12 Horiguchi M, Nagata S, Yamamoto N, et al. Kinetics of indocyanine green dye after intraocular surgeries using indocyanine green staining. Arch Ophthalmol 2003;121:327-31.

13 Tadayoni R, Paques M, Girmens JF, et al. Persistence of fundus fluorescence after use of indocyanine green for macular surgery. Ophthalmology 2003; 1 10:604-8

14 Stalmans $\mathbf{P}$, Van Aken EH, Veckeneer $M$, et al. Toxic effect of indocyanine green on retinal pigment epithelium related to osmotic effects of the solvent. Am J Ophthalmol 2002;134:282-5.

15 Ho JD, Tsai RJF, Chen SN, et al. Toxic effect of indocyanine green on retinal pigment epithelium related to osmotic effects of the solvent. Am J Ophthalmol 2003; 135:258; author reply 259 .

16 Bhisitkul RB. Second generation vital stains in retinal surgery. Br J Ophthalmol 2003;87:664-5.

17 Li K, Wong D, Hiscott P, et al. Trypan blue staining of internal limiting membrane and epiretinal membrane during vitrectomy: visual results and histopathological findings. Br J Ophthalmol 2003;87:216-19.

18 Teba FA, Mohr A, Eckardt C, et al. Trypan blue staining in vitreoretinal surgery. Ophthalmology 2003;1 10:2409-12.

19 Meyer CH, Rodrigues EB, Kroll P. Trypan blue has a high affinity to cellular structures such as epiretinal membrane. Am J Ophthalmol 2004;137:207-8; author reply 208.

20 Veekeneer M, van Overdam K, Monzer J, et al. Ocular toxicity study of trypan blue injected into the vitreous cavity of rabbit eyes. Graefes Arch Clin Exp Ophthalmol 2001;239:698-704.

21 Stalmans P, Van Aken EH, Melles G, et al. Trypan blue not toxic for retinal pigment epithelium in vitro. Am J Ophthalmol 2003;135:234-6.

22 Haritoglou C, Gandorfer A, Gass CA, et al. Indocyanine green-assisted peeling of the internal limiting membrane in macular hole surgery affects visual outcome: a clinicopathologic correlation. Am J Ophthalmol 2002;134:836-41.

23 Sheidow TG, Blinder KJ, Holekamp N, et al. Outcome results in macular hole surgery: An evaluation of internal limiting membrane peeling with and without indocyanine green. Ophthalmology 2003;110:1697-701.

24 Ando F, Sasano K, Ohba N, et al. Anatomic and visual outcomes after indocyanine green-assisted peeling of the retinal internal limiting membrane in idiopathic macular hole surgery. Am J Ophthalmol 2004;137:609-14.

25 Gandorfer A, Messmer EM, Ulbig MW, et al. Indocyanine green selectively stains the internal limiting membrane. Am J Ophthalmol 2001;131:387-8.

26 Perrier M, Sebag M. Trypan blue-assisted peeling of the internal limiting membrane during macular hole surgery. Am J Ophthalmol 2003;135:903-5. 
27 Feron EJ, Veckeneer M, Ginderdeuren RPV, et al. Trypan blue staining of epiretinal membranes in proliferative vitreoretinopathy. Arch Ophthalmol 2002;120:141-4.

28 Kwok AKH, Lai TYY, Man-Chan W, et al. Indocyanine green assisted retinal internal limiting membrane removal in stage 3 or 4 macular hole surgery. Br J Ophthalmol 2003;87:71-4.

29 Kwok AKH, Lai TYY, Yuen KSC, et al. Macular hole surgery with or without indocyanine green stained internal limiting membrane peeling. Clin Experiment Ophthalmol 2003;31:470-5.
30 Kadonosono $\mathrm{K}$, Itoh N, Uchio $\mathrm{E}$, et al. Staining of internal limiting membrane in macular hole surgery. Arch Ophthalmol 2000;118:1116-18.

31 Da Mata AP, Burk SE, Riemann CD, et al. Indocyanine green-assisted peeling of the retinal internal limiting membrane during vitrectomy surgery for macular hole repair. Ophthalmology 2001;108:1187-92.

32 Ben Simon GJ, Desatnik H, Alhalel A, et al. Retrospective analysis of vitrectomy with and without internal limiting membrane peeling for stage 3 and 4 macular hole. Ophthalmic Surg Lasers Imaging 2004;34:109-15.

\section{Register now!}

10th European Forum on Quality Improvement in Health Care

13-15 April 2005, ExCel Conference Centre, London

For further information on how to register please go to:

http://www.quality.bmipg.com 